\title{
The Use of Electron Ptychography to Implement Efficient Phase Imaging in STEM
}

\author{
P D Nellist ${ }^{1}$, H Yang ${ }^{1 *}$, L Jones ${ }^{1}$, G T Martinez ${ }^{1}$, R N Rutte ${ }^{2}$, B D Davis ${ }^{2}$, T J Pennycook ${ }^{3}$, M Simson ${ }^{4}$, \\ M Huth ${ }^{4}$, H Soltau $^{4}$, L Strüder $^{5}$, R Sagawa ${ }^{6}$ and Y Kondo ${ }^{6}$ \\ 1. Department of Materials, University of Oxford, Parks Rd, Oxford UK. \\ 2. Department of Chemistry, University of Oxford, 12 Mansfield Road, OX1 3TA, Oxford UK. \\ 3. Department of Physics, University of Vienna, Boltzmanngasse 5, 1090 Vienna, Austria. \\ 4. PNDetector GmbH, Sckellstraße 3, 81667 München, Germany. \\ 5. PNSensor GmbH, Otto-Hahn-Ring 6, 81739 München, Germany. \\ 6. JEOL Ltd.,3-1-2 Musashino Akishima Tokyo 196-8558 Japan. \\ * Now at Molecular Foundry, Lawrence Berkeley National Laboratory, Berkeley, CA, 94720, USA.
}

Historically, the scanning transmission electron microscope has not been widely used for phase contrast imaging because the small bright-field detector required detects only a small fraction of the incident electrons and is therefore inefficient with respect to dose. This limitation has hindered the efficient imaging of light elements in STEM. Alternative modes also have limitations. For example, annular dark-field (ADF) imaging of graphene only makes use of a few percent of the incident electrons, and annular bright-field imaging $(\mathrm{ABF})$ requires lens aberrations to form an effective phase plate to get contrast from weakly scattering objects.

Electron ptychography in the STEM was first demonstrated more than 20 years ago in the context of improving image resolution [1]. At that time, the image field of view was restricted by the limitations of the camera technology. Here we make use of the pnCCD (S)TEM camera, a direct electron pixelated detector from PNDetector, mounted on the JEOL ARM200-CF aberration corrected microscope. The detector has a grid of $264 \times 264$ pixels and operates at a speed of 1000 frames-per-second (fps). The detector can achieve a speed of up to 20,000 fps through binning/windowing. ADF images can be recorded simultaneously, as shown by the schematic in Fig. 1.

The resulting 4D data set is formed of a series of coherent convergent beam diffraction patterns recorded as a function of illuminating probe position. This data set is a very rich source of information. By adapting a previously used method [2] for use with a modern aberration-corrected STEM [3], high quality, quantitative phase images can be formed simultaneously with other STEM imaging modes such as ADF. The method operates entirely in reciprocal space where specific spatial frequencies in the reconstructed phase image are known only to arise from specific locations in the detector plane (Fig. 2). This phase imaging mode has a relatively simple transfer function [4] and also provides an inherent filter of image noise without reducing the signal strength to form high quality phase images (Fig. 3). It is also shown that residual aberrations in the image can be detected and corrected. Three dimensional information is also available post-acquisition by reconstructing the phase image at a specific depth in the sample, which can be performed even though the microscope may not have been focused at that depth.

It is also found that the phase images formed through electron ptychography can also be relatively intuitively interpreted in thicker, heavier samples where dynamical electron diffraction dominates. In all oxide materials imaged to date, all the cations and the $\mathrm{O}$ columns are clearly visible. We also explore from a theoretical point why ptychography shows a robustness to dynamical scattering compared to conventional phase contrast imaging. 
Finally we explore how pixelated detectors can play a role in the dark-field scattering region of the STEM detector plane to detect the influence of strain and variations in composition [5].

[1] P.D. Nellist, B.C. McCallum and J.M. Rodenburg, Nature 374 (1995) 630-632.

[2] J.M. Rodenburg, B.C. McCallum and P.D. Nellist, Ultramicroscopy 48 (1993) 303-314.

[3] T.J. Pennycook et al., Ultramicroscopy 151 (2015) 160-167.

[4] H. Yang et al., Ultramicroscopy 151 (2015) 232-239.

[5] The authors acknowledge funding from the EPSRC through grant number EP/M010708/1.

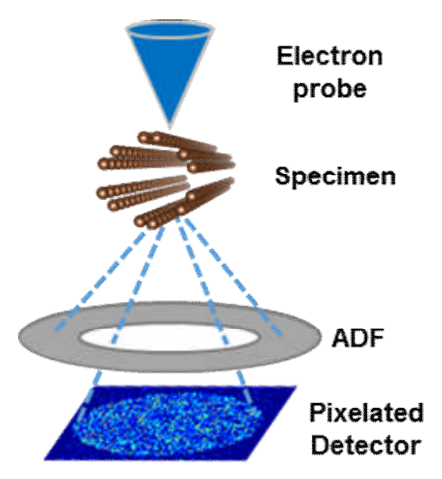

Figure 1. A schematic diagram of the STEM showing electron scattering being detected by an $\mathrm{ADF}$ detector and a fast pixelated camera simultaneously.

Figure 2. For a specific spatial frequency in the reconstructed phase image, the information can be derived from interference observed in the detector plane leading to a map of the amplitude (left) and phase (right) associated with the
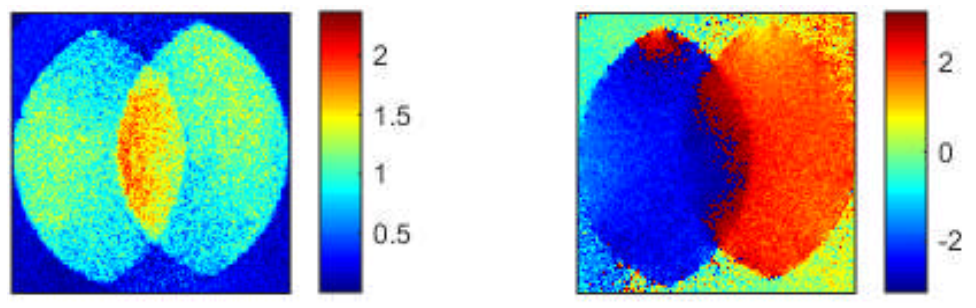
spatial frequency.
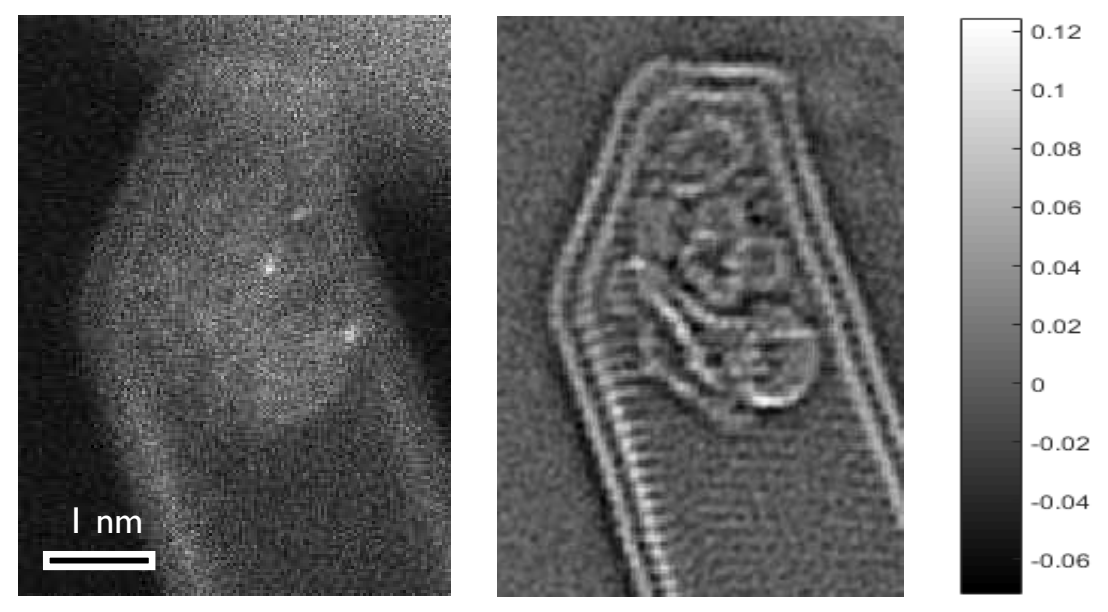

Figure 3. Simultaneous ADF (left) and phase imaging (right) of a double-wall carbon nanotube containing a carbonaceous material doped with iodine atoms. The iodine atoms are clearly visible in the ADF image. The lattice in the walls of the carbon nanotube are visible in the phase image. The greyscale of the phase image is shown with units of radians. 